\title{
Research on Mars Communication Link Budget
}

\author{
Hao Wu ${ }^{1, a^{*}}$, Chenghua Wang ${ }^{1, b}$ and Jie Wang ${ }^{1, c}$ \\ ${ }^{1}$ Nanjing University of aeronautics and astronautics electronic Information engineering college \\ Nanjing 211100, China
}

awuhao0408102@foxmail.com,bchwang@nuaa.edu.cn, cwj1391992076@163.com

\begin{abstract}
Keywords: Mars communication; Communication channel; Link budget; Solar scintillation; Rainfall attenuation
\end{abstract}

\begin{abstract}
This paper presents the budget method of the Mars-earth communication link. The calculation models of Mars atmosphere loss, Mars sandstorm loss, solar scintillation loss, earth atmosphere absorption; rainfall loss and hot body noise are studied. The propagation characteristics of electromagnetic waves in Mars atmosphere are analyzed and modeled by the characteristics of the propagation of analogous waves in the Earth's atmosphere. Ultimately, we found that the surface of the radio waves propagation environment is ideal, with less attenuation loss. Especially, the calculate model of solar scintillation loss and rainfall loss is simulated, and the simulation results are consistent with the actual statistical results, which proves the rationality of the calculate model. At last, an example of Mars-earth communication link budget is given. With the procedure, we can get one of the most important parameters of the link-- Carrier to Noise Ratio, and we know the bit signal to noise ratio required by the right BER, then the maximum code rate of the link can be got. This paper has some reference to the design of Mars communication link.
\end{abstract}

\section{Introduction}

In the process of Mars detection, the deep space communication link is the link between the ground station and deep space probe, only to ensure the smooth flow of communication links, it may make the success of Mars exploration mission. Because the communication distance of Mars-Earth communication is long and the communication environment is bad, the design of communication system will be restricted. Only by studying the channel characteristics, the system plan and optimize of the Mars-earth communication link can be executed, and the key of designing a communication system is link budget and channel simulation. At the present stage, the main research in deep space communication focused on the lunar-earth communications and the moon surface communications, and research on the Mars-earth and Mars surface communication link budget is much less. In literature [1], the launch cruise and EDL (Entry, Down, Land) of the Mars Science Laboratory are introduced systematically. A more complete lunar-earth communication link budget method is given in literature [2]. In literature [3, 4], the solar scintillation of Mars communication was studied deeply. The radio propagation characteristics on the surface of Mars were studied in literature [5], and the calculation model of near-earth space rainfall attenuation was given in literature [6]. Literature [7] and [8] analysis the influence of the receiving antenna and cosmic noise on the communication performance. This paper summarizes the above research results, studies the Mars-Earth communication link budget method, and builds a link budget program with Microsoft Foundation Classes.

\section{Communication Link Analysis}

Mars-earth Communication Link. There are two types of communication between Mars detectors and earth stations. One is Direct to Earth (DTE) and the other is communication with earth stations through Mars relay satellites. For the deep space transmission link, we can divide it into three parts: the near-earth space propagation link, the interplanetary space propagation link and the target star space propagation link. We should consider the influence of the atmospheric and electromagnetic environments around the earth and the target star on the propagation of the signal. The interstellar 
space propagation link can be regarded as the propagation of the signal in free space.

Communication Link Budget Formula. Assuming an ideal communication system, there is no loss in the components of the system, the transmission medium is free space, the transmit antenna gain is $G_{T}$, the receiving antenna gain is $G_{R}$, the transmit power is $P_{T}$, the receiving power is $P_{R}$, the communication distance is $\mathrm{d}$, the carrier frequency is $\mathrm{f}$, the wavelength is $\stackrel{\lambda}{\text {, the receiving }}$ antenna power is

$$
P_{R}=P_{T} G_{T} G_{R}\left(\frac{\lambda}{4 \pi d}\right)^{2}
$$

If $L_{f}$ is the free space link loss, the formula can be transformed into

$$
P_{R}=\frac{P_{T} G_{T} G_{R}}{L_{f}}
$$

If we use the form of decibels, that is

$$
P_{R}=P_{T}+G_{T}+G_{R}-L_{f}
$$

There will be a variety of losses and a variety of noise interference in signal propagation. In communication link analysis, the primary concern is the carrier-to-noise ratio at the input of the receiver in the communication system between the spacecraft and the earth station or between the spacecraft and the spacecraft [9]. Usually $\underline{C / N}$ is used to represent the carrier-to-noise at the input of the receiver.

Because the receiver system equivalent noise power is

$$
N=k T_{S R} B_{N}
$$

And we use decibels as unit, the carrier-to-noise ratio is

$$
C / N=E I R P+\left(G_{R} / T_{S R} L_{F}\right)-k-B_{N}-L(d B)
$$

Wherein

$$
\frac{G_{R}}{T_{S R} L_{F}}=\frac{G_{R r}}{T_{S R}}=\frac{G_{R}}{T_{S}}=\frac{G}{T}
$$

Consider the logarithm of the Boltzmann constant, then

$$
k=10 \log \left(1.38 \times 10^{-23}\right)=-228.6(\mathrm{dBW} / \mathrm{kHz})
$$

Eq. 5 can be converted to

$$
C / N=E I R P+(G / T)-B_{N}-L(d B)+228.6(d B)
$$

Eq. 8 is the basic link budget formula.

\section{Mars-earth Communication Link Budgeting}

The Mars-earth communication link budget mainly considers two aspects which is carrier signal loss and noise increase. The carrier signal loss mainly includes Mars atmospheric loss, Martian sandstorm effect, solar scintillation loss, free space loss, Earth's atmospheric loss, and the noise increase is mainly cosmic noise and receiver thermal noise.

Near Space Propagation Link of Mars. Mars is the fourth planet of the solar system, and its atmospheric composition is quite different from the Earth's. Martian atmosphere is relatively thin, 
because Mars is only a quarter of the Earth's mass. The main components of the atmosphere are 95 percent carbon dioxide, 3 percent nitrogen, 1.6 percent hydrogen and traces of oxygen, water vapor and methane. Because of the low water content in the Martian atmosphere, Mars water can only frost on the surface, it is impossible to rain, so we needn't consider the impact of Mars water vapor on electromagnetic waves, and carbon dioxide absorption is micron level, so it has almost no effect on the link when we use X-band. In general, the atmospheric absorption of Mars almost no effect on the link, but the sandstorm on Mars will have a great impact on the communication link. However, the influence of dust weather on electromagnetic waves is very complex. Meanwhile, the static electricity from Mars dust can cause large-scale and high-intensity discharge phenomena on communication equipment, and high-speed movement of sand particles is likely to cause serious damage to communication equipment. Therefore, it is recommended that communication be carried out under conditions of non-sandstorms or weak dust storms.

Free Space Loss. The electromagnetic wave loss due to the transmission distance can be regarded as the free space loss, as Eq.9.

$$
\mathrm{L}_{f}=20 \log f+20 \log d+92.442
$$

Wherein, $d$ is transmit distance, the unit is $\mathrm{km}, f$ is frequency, the unit is $\mathrm{MHz}$

Solar Scintillation Loss. Planetary polymerization consists of two types: superior conjunction and inferior conjunction. In superior conjunction, the sun is between Earth and Mars, so the received signal is weakest [4], and the intensity of Mars detection radio signal flicker occurs because of the solar wind or solar corona effects. The signal intensity fluctuation is usually expressed by the scintillation factor $\mathrm{m}$. It is generally considered that it is a weak scintillation when the scintillation index $\mathrm{m}$ is less than 1 . In weak solar scintillation, the transmission channel can be approximated as a Rican fading channel [10]. The relationship between the Rican factor $\mathrm{K}$ and the scintillation index is as follows.

$$
\mathrm{K}=\frac{\sqrt{1-m^{2}}}{1-\sqrt{1-m^{2}}}
$$

Atmospheric Attenuation. Radio waves pass through the atmosphere in the near-earth space, the atmosphere changes constantly led the receivers receive level fluctuation changes, resulting in signal fading atmospheric fading generally divided into the following types [6]: fading due to clouds and rain, fading due to the absorption of electromagnetic energy by water molecules and oxygen molecules, atmospheric tropospheric multipath fading, and atmospheric ionosphere fading. ITU has given the above fading type calculation model $[11,12]$. Since the calculation method of fog fading and water molecule and oxygen molecule absorption fading is similar to the calculation of rainfall fading, we only introduce the calculation method of rainfall fading, we establish the rain fading model according to the following steps.

According to the rainfall intensity of the ground station $\operatorname{rain}^{R_{p}}$, we can get the rain attenuation rate $\gamma_{R}(\mathrm{~dB} / \mathrm{km})$.

$\gamma_{R}=K \cdot R_{p}^{\alpha}$

Wherein, $\mathrm{K}=4.21 \times 10^{-5} \times f^{2.42}, \alpha=1.41 \times f^{-0.0779}$.

Find the rain height $h_{R}(\mathrm{~km})$, altitude $\mathrm{H}(\mathrm{km})$, latitude $\varphi$ and elevation angle of the ground station antenna $\theta_{R}$, and calculate the equivalent path length $L_{S}$.

$\mathrm{h}_{\mathrm{R}}=4.0-0.075(\varphi-36)$

$L_{S}=\left(\mathrm{h}_{\mathrm{R}}-H\right) / \sin \theta_{R}$

Then we calculate the area rainfall loss $\mathrm{L}(\mathrm{dB})$ :

$\mathrm{L}=\gamma_{R} \times L_{S}$

Cosmic Noise. At present, the only way to calculate solar noise is measured. For example, according to the United States Goethe Salmo deep space station with high efficiency antenna [13], 
in 1987 (at the beginning of the solar cycle) $\mathrm{X}$ band measured data obtained from the sun noise temperature curve expression is

$$
T_{\text {sun }}=800 e^{-2.0 \theta}
$$

Wherein, $\underline{\theta}$ is the angle between the center of the detector beam and the center of the solar plane.

The maximum noise temperature of the moon is

$$
T_{\text {moon }}=T_{b} \times 0.90 \times \eta_{\text {ant }}
$$

Wherein, $T_{b}=240 \mathrm{~K}$, and $\eta_{\text {ant }}$ is antenna efficiency, and it's typically 0.70 to most large antennas.

\section{Mars-earth Communication Link Budget Simulation}

Simulation Analysis of the Effect of Solar Scintillation on Link. MATLAB is used to simulate the effect in weak solar scintillation. Fig. 1 show the bit error rate when the scintillation index $\mathrm{m}$ takes $0,0.1,0.2$ and 0.3. Fig. 2 show the effect of solar scintillation on bit error rate when the solar-earth-probe angle (SEP) is $2.3^{\circ}$ and the X-band and KA-band corresponding to the solar scintillation index, respectively, 0.37 and 0.06 .

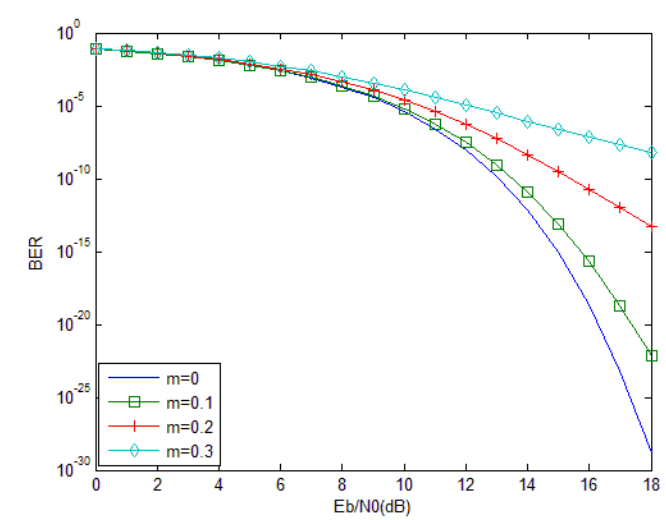

Figure 1. Communication link performance At different scintillation index

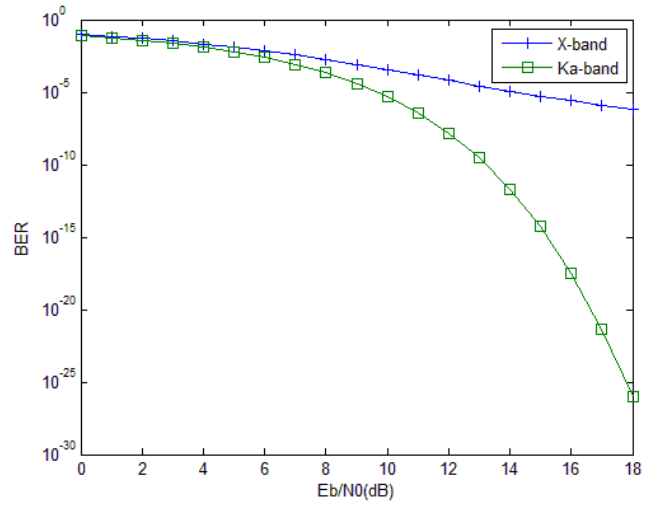

Figure 2. Effect of solar scintillation on communication link performance at $\mathrm{X}$ and Ka-band

Mars-Earth communication link bit error rate requirements are generally $10-5$, to meet this bit error rate requirements, the communication link need more $2 \mathrm{~dB}$ when $\mathrm{m}$ is 0.3 than when $\mathrm{m}$ is 0 . We can see in Fig. 2 that the same solar scintillation the X-band signal to improve the bit error rate is much, the sun scintillation effect on Ka-band is still relatively small, that is, the higher the frequency, the stronger the ability of anti-solar scintillation.

Simulation Analysis of Influence of Rainfall on Link. According to the rainfall fading model established in the previous paper, the rainfall loss of the communication link under different elevation angles of the ground station and the different rainfall rate is obtained, as shown in Fig. 3.

From the simulation results, it can be seen that the greater the rainfall rate is, the greater the attenuation is. The lower the elevation angle of the antenna is, the greater the rainfall attenuation is; in the same situation, the rainfall attenuation of the Ka-band is much higher than that of the X-band. The simulation results are consistent with the actual statistical results. 

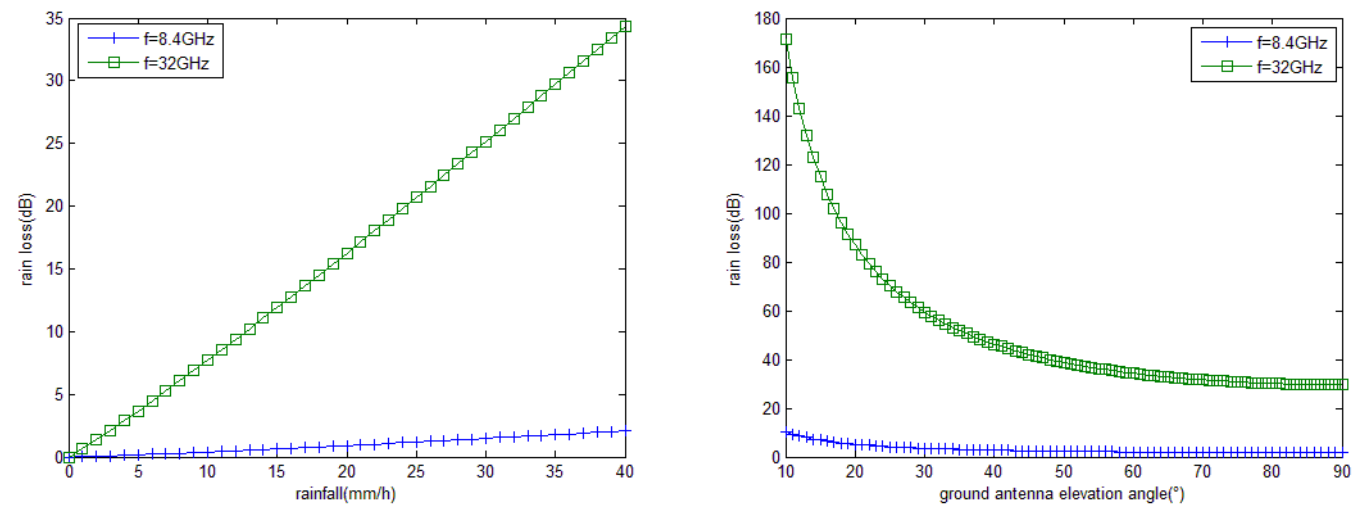

Figure 3. Rain attenuation under different conditions

\section{Communication Link Budget Software}

The software interface is shown in Fig. 4, enter the query parameters, you can get the link budget results, including the maximum rate under the required bit error rate.

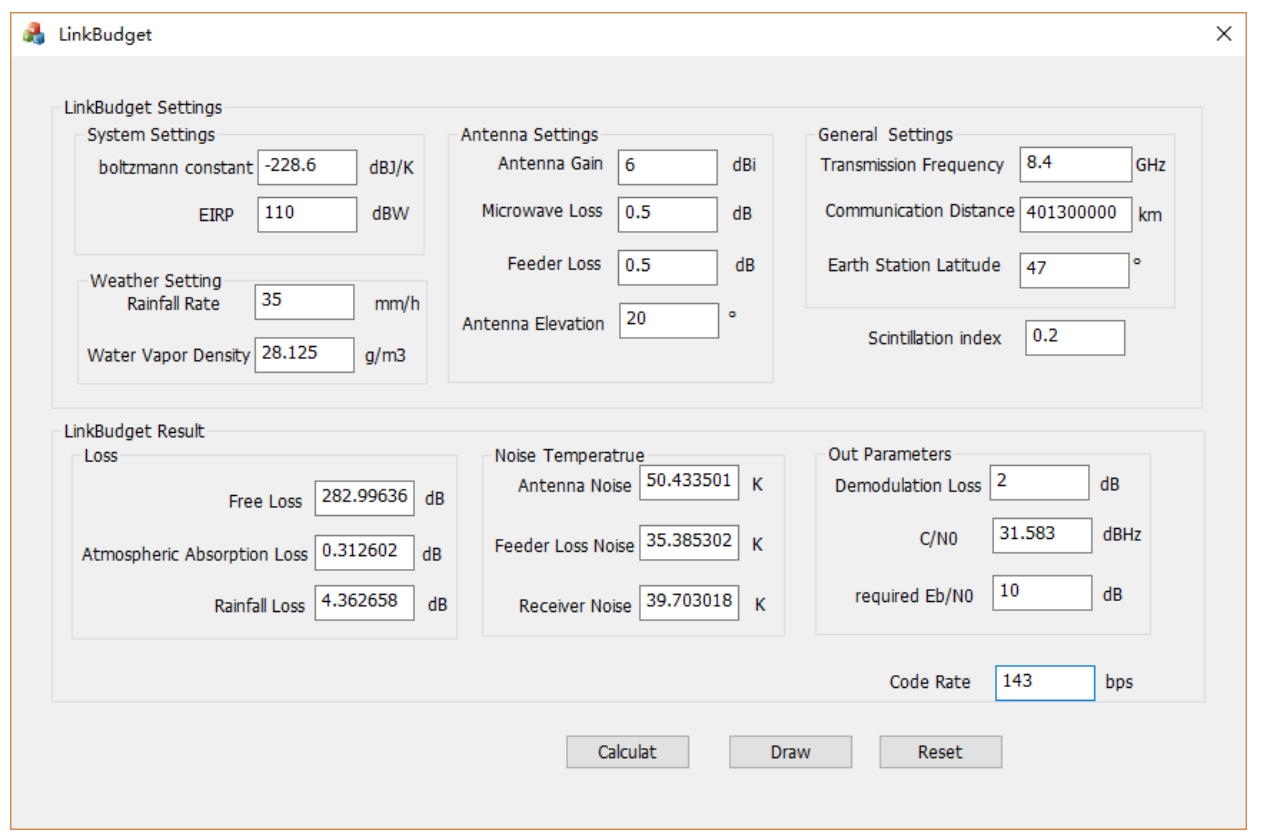

Figure 4. Mars-earth communication link budget software

\section{Summary}

In this paper, the link budget is established for Mars-earth communication. The communication channel is analyzed. The calculation models of the atmosphere loss, the solar scintillation loss, the earth atmosphere absorption loss, the rainfall loss, the snowfall loss and the thermal body noise are studied. This paper has some reference significance for studying the propagation characteristics of Mars-earth and the deep space communication system

\section{References}

[1] A. Makovsky, P. Ilott, J. Taylor: DESCANSO_Design and Performance Summary Series: Mars Science Laboratory Telecommunications System Design (Jet Propulsion Laboratory California Institute of Technology, America, 2009), p. 100..

[2] X. Y. Wang, C. H. Wang and Q. M. Zhu: Internet Technology and Applications, 2011 International Conference on. IEEE, 2011: 1-4. 
[3] D. Morabito and R. Hastrup: Aerospace Conference Proceedings (2002), Vol. 3, p. 1271.

[4] T. Wu, Y. Yan, Y. Li, C.M. Wang, X.J. Yao and X. Li: 2014 Fourth International Conference on Instrumentation and Measurement, Computer, Communication and Control (Harbin, China, September, 2014), 2014, p.510.(In Chinese).

[5] N. Zhao, X. Meng and S. B. Hu: Journal of Space Science, Vol. 29 (2009) No.2, p.244 (In Chinese).

[6] ITU, Specific Model of Rain Attenuation Used in Prediction, Rec ITU-R, 2005, p.838-3.

[7] Z. Li, M. Fan and H. T. Li: Journal of Spacecraft TTC\&C Technology, Vol. 28 (2009) No.6, p.15 (In Chinese).

[8] A. K. Kundu, M. T. H. Khan and W. Sharmin: Electrical Information and Communication Technology (2013), 2014, p.1.

[9] I. F. Akyildiz, O. B. Akan, and C. Chen: Computer NetWorks, Vol. 43 (2003) No.2, p.75.

[10] A. Hooke: Communication of the ACM, Vol. 44 (2001) No.9, p.38.

[11] ITU, Attenuation by atmospheric gases. ITU-R, 2012, p.676.9.

[12] ITU, Attenuation due to clouds and fog. ITU-R, 2012, p.840-5.

[13] J. Wang: Deep Space Communication Channel Model Research and Simulator Implementation for Mars Mission (MS., Beijing Institute of Technology, China, 2015), p.14. (In Chinese). 